\title{
Beta Blockers Use in Cardiac Failure: Does the Current Prescribing Practice at a Large Urban Hospital in Zimbabwe Exhibit Evidence Based Care and Offer Optimal Therapy for Cardiac Failure Patients?
}

\author{
Patrick Rutendo Matowa \\ Pharmaceutical Technology Department, Harare Institute of Technology, Harare, Zimbabwe \\ Email: patmat01@yahoo.co.uk
}

Received 1 April 2015; accepted 25 May 2015; published 28 May 2015

Copyright (C) 2015 by author and Scientific Research Publishing Inc.

This work is licensed under the Creative Commons Attribution International License (CC BY). http://creativecommons.org/licenses/by/4.0/

(c) $\underset{\mathrm{EY}}{\mathrm{F}}$ Open Access

\begin{abstract}
Background: Cardiac failure treatment largely focused on symptomatic relief at the expense of the address of the underlying disease process of cardiac remodelling. This old wisdom of practice has been turned around by clinical research findings that have shown that there are agents that reverse cardiac remodelling and offer long-term benefits to cardiac failure patients. This has led to the recommendation of evidence-based practice in chronic heart failure management using reverse modelling agents such as beta blockers. Objectives: To ascertain the prescribing patterns of beta blockers in cardiac failure patients by doctors in a public hospital setting and determine the prevalence of cardiac failure hospitalisation and the age groups involved. Study design: A retrospective medical records review observational study. Methodology: A sample size of $\mathbf{3 8 5}$ cardiac failure cases was used. Data on cardiac failure patients who were once hospitalised at the hospital of study were abstracted from the patients' medical records files using data collection forms. Results: There were $36(9.4 \%)$ patients who were prescribed beta blockers, 7 patients had their beta blocker substituted for another. Atenolol was prescribed to $30(7.8 \%)$ patients, propranolol to 7 $(1.8 \%)$ and carvedilol to $6(1.6 \%)$ patients. Metoprolol and bisoprolol were not prescribed at all. There were more females $(57.9 \%)$ than males $(42.1 \%)$ and the mean age was 41.9 (standard deviation 24.0) years. The prevalence of cardiac failure hospitalisation was $1.54 \%$. Conclusion: The rate of beta blocker prescribing was low. There is need for emphasis on evidence-based treatment options in the management of cardiac failure in Zimbabwe.
\end{abstract}


Keywords

Cardiac Failure, Beta Blockers, Prescribing Patterns

\section{Introduction}

Beta blockers are indicated in the following types of heart failure; systolic heart failure, diastolic heart failure and atrial fibrillation. Heart failure therapy has traditionally concentrated largely on symptomatic relief rather than addressing the underlying disease processes. The disease processes are engulfed in cardiac remodelling. Cardiac remodelling encompasses many changes associated with progressive heart failure. Therapeutic interventions aimed at solely correcting a low cardiac output or reduced blood flow; those offering symptomatic relief or improved cardiac emptying, do not necessarily slow heart failure progression or reduce mortality [1]-[4]. Prevention of the progression of heart failure by reversing the remodelling process should be the target for therapy, in addition to improving symptoms and reducing morbidity and mortality [1] [5]. Systems and factors that influence remodelling where therapy can be targeted towards, include the renin angiotensin system, sympathetic nervous system, endothelin, cytokines (Tumor Necrosis Factor, TNF, and interleukins) and nitrous oxide and oxidative stress. Large randomized studies have assessed measures of remodelling that include ejection fraction, left ventricular end-diastolic volume and left ventricular systolic volume, which are important in heart failure progression [1].

Traditionally, beta blockers were avoided in heart failure management. The rationale being that, the sympathetic nervous system over-activity provided an important compensatory protection for the failing heart. Thus blocking this level of compensation using a beta blocker would risk precipitating or worsening heart failure [6] [7].

Subsequent studies and clinical trials have challenged this old school of thought and practice [6] and have demonstrated that beta blockers reduce mortality and morbidity [8] [9]. Although the risks remain, there is a need to strike a balance between those short term risks and long term benefits of using beta blockers. At first, patients may feel worse from the blockade of the sympathetic nervous system activity, thus the need for starting with low doses and gradually increasing to the optimum dose [6]. Multiple clinical trials have proved the benefits of beta blockade in cardiac failure [6]. In all these studies, the beta blockers were administered in conjunction with an angiotensin converting enzyme inhibitor and a diuretic(s). Beta blockade consistently improved left ventricular function and provided additional clinical benefits over and above those achieved on standard therapy alone (i.e. Angiotensin converting enzyme inhibitor, ACEI, + diuretic) [6] [10]. Beta blockers reduce morbidity and mortality tremendously, mainly due to reversal of cardiac remodelling which subsequently would improve ejection fraction, left ventricular function and geometry [1] [6] [11]. The trials have shown overwhelming benefits of beta blockers in heart failure, such that the Cardiac Insufficiency Bisoprolol Study II, CIBIS-11, and the Metoprolol CR/XL Randomised Intervention Trial in congestive Heart Failure, MERIT-HF, studies were ended prematurely due to pronounced benefit in the treatment groups. Benefits from beta blockers include improved survival (30\% - 35\%), reduced need for hospitalisation and reduced symptoms of heart failure [6].

The main aim of beta blockade in chronic heart failure is not short term relief of symptoms but improvement in LV function and long-term outcomes. The long term benefits of beta blockers in heart failure include improved survival, improved control of heart failure, reduced need for hospitalisation, improved quality of life and improved left ventricular ejection fraction [6]. The short term risks of beta blockers in heart failure are worsening heart failure, bradyarrhythmias, prolonged intra-ventricular conduction, hypotension and worsening renal function [6]. These short term risks must be balanced against the long term benefits of using beta blockers in heart failure. Their mechanisms in improving survival in heart failure include antiarrhythmic action, anti-ischaemic action, attenuation of catecholamine toxicity and reduced cardiac remodelling [6].

The use of beta blockers in heart failure has been shown to significantly reduce mortality and hospital admissions by approximately a third. Since heart failure carries a high risk of death and disability, these important benefits of beta blockers should be utilised as a priority by clinicians in the management of heart failure [12].

\subsection{Research Problem}

Due to rapid urbanisation, dietary changes, lifestyle modifications, risk-prone behaviour and cultural attitudes 
predominantly in young people in Africa; prevalence of heart failure is on the rise substantially in sub-Saharan Africa [13]. Heart failure is emerging as a dominant form of cardiovascular disease and has massive social and economic relevance due to its high prevalence, mortality, morbidity and negative impact on young economically active individuals. The cases of heart failure in Africans are largely (about 90\%) due to hypertension, cardiomyopathy, heumatic diseases, chronic lung diseases and pericardial disease; and remaining largely non-ischaemic [13].

In the abundance of all this overwhelming evidence of the rise in heart failure cases coupled with the discoveries of more effective beta blockers, the Zimbabwean health care system still finds itself stuck with the less effective beta blockers at the expense of the proven ones from many clinical studies. The Zimbabwe national treatment guidelines, Essential Drug List of Zimbabwe (EDLIZ), has no updated recommendations on the use of beta blockers in heart failure in line with new international guidelines which advocate for the use of the more efficacious beta blockers such as carvedilol and metoprolol rather than the outdated and less effective atenolol and propranolol. In as much as the concept of the EDLIZ is appreciated, it is the manner and period it is reviewed that, sometimes, leaves the health care provision disabled. This is so when lifesaving medicines like the newer beta blockers, are overlooked during the review of the treatment guidelines in the gist of them "being expensive or unaffordable”. Therefore, lack of evidence based clinical guidelines for the management of heart failure using beta blockers in Zimbabwe is of major concern.

Moreover, current evidence suggests that some primary care physicians underperform in their management of heart failure patients [7] [14]. While most doctors prescribe medication appropriately, some doctors display patterns that are inconsistent with evidence-based medicine [14]. Identifying those patterns can offer intervention opportunities that might otherwise remain unnoticed. Heart failure is a chronic condition that poses a major growing concern, negative impact and threat to the public and country's economy; especially during this era of Human Immunodeficiency Virus (HIV) pandemic. HIV is an important cause of heart failure in most African countries. HIV cardiac related pathology, mainly dilated cardiomyopathy (CM) and TB pericarditis, is on the rise and poses a serious threat to the health care systems in Africa [15].

\subsection{Significance of the Study}

In order to engage in meaningful economic activities, people have to be in a good and sound state of health. Thus, improving the effectiveness of care and optimising patient outcomes through utilisation of evidence based practice becomes increasingly important.

The study will help identify the prescribing patterns of beta blockers in the public health sector and subsequently indicate the areas the country's health care is faring well and where amendments or updates are necessary, in terms of beta blocker therapy in chronic heart failure patients. This study would help in the formulation of clinical guidelines on how to manage heart failure patients using beta blockers, improving the knowledge of clinicians on the use of beta blockers in heart failure cases and optimisation of beta blockers in heart failure.

\section{Aims of Study}

The purpose of this study is to establish the prescribing patterns of beta blockers in chronic heart failure patients in one of Harare's major public hospitals; in order to get a picture of whether heart failure patients are receiving optimal evidence based therapy in line with clinical studies supporting the use of certain beta blockers in heart failure. This would help in policy implementations such as the formulation of clinical guidelines for the management of heart failure using beta blockers and also continual education of clinicians on the importance and use of beta blockers in heart failure.

\section{Objectives of Study}

1) To assess the prescribing patterns of beta blockers in heart failure patients by doctors in a large urban public hospital, in Harare, and compare against evidence from clinical trials.

2) To identify disease conditions that are common in heart failure.

3) To ascertain the prevalence of heart failure hospitalisations and the age groups involved, at the hospital.

4) To determine the most prescribed beta blockers in heart failure cases at the hospital. 


\section{Methods}

\subsection{Study Design}

An observational retrospective medical records review study

\subsection{Study Tool and Data Collection}

Data collection forms were constructed and used by the investigator to abstract information from heart failure patients' medical files.

\subsection{Study Setting and Population}

One of Harare's major public referral hospitals was used for the study. A total of 385 heart failure cases, as calculated using Cochrane's sample size formula, were identified and studied.

\subsection{Inclusion and Exclusion Criteria}

The study included all heart failure cases which fell within the sample size, retrospectively from December 2011, and excluded all non-heart failure cases. Age was not taken into account

\subsection{Sample Size Calculation}

The sample size was calculated using Cochrane sample size formula for a single proportion.

$$
n=(z / \Delta)^{2} \times p(1-p)
$$

where, $n$ is sample size;

$z$ is 1.96 (from statistical tables at $95 \%$ confidence interval);

$\Delta$ is level of precision $( \pm 5)$;

$p$ is the estimated proportion of an attribute that is present in the population or degree of variability (50 in this case).

This yielded a sample size of 385 .

\subsection{Sampling Procedure}

All heart failure cases of the year 2011 (317 cases) and some from the year 2010 (68 cases) were picked up for study without any form of order or systematic random selection method employed, until the sample size of 385 cases was attained.

\subsection{Data Collection Procedure}

The hospital medical records department was used as the source for the heart failure cases. Data collection forms were used to capture the relevant information from the selected medical files. Data collected included gender, age, date of admission and discharge, diagnosis, related disease conditions, medical history, whether the patient was on a beta blocker prior to admission, any beta blocker prescribed upon admission, beta blocker dosing schedule, other heart failure medication taken by the patient, cardiovascular examination (ECG, ECHO), any other medical tests done and reason for discharge.

\subsection{Ethical Considerations}

Authorisation from Joint Research Ethical Committee, JREC, to carry out the study, and also from the Medical Records department, to have access to patient medical records, was sought for and granted.

All patient identification information was not recorded onto the study data collection forms.

\subsection{Data Analysis and Model Procedure}

Data was statistically analysed using Epi Info package for 1) the frequencies of a particular gender, beta blockers prescribed, associated diseases, death and discharge 3) for the association between disease exposure and beta 
blocker prescription

Statistical Package used was R software package version 2.15.1 (2013). The modelling environment applied in the statistical analysis aimed to establish if association exists between: 1) disease an individual is exposed to and the physician beta blockers prescribing pattern (prescribe/do not prescribe); and 2) the treatment (standard + beta blockers (BB) or standard + other drugs) and the occurrence of death. An ACEI (enalapril/captopril) and a loop diuretic (furosemide) were used as the standard treatment in the study. The outcome of interest in both cases was binary, making a logistic regression a suitable approach in predicting the outcome.

The model procedure follows a stepwise regression analysis approach. With the stepwise approach we begin by bivariate analysis, to test for association between single exposure variable and outcome of interest.

The prevalence of heart failure hospitalisation was calculated from the total hospitalisations of the year 2011; data was obtained from the medical records department.

\section{Results}

The results show that there were less males (42.1\%) than females (57.9\%) hospitalised with heart failure, as shown in Table 1 below.

The average age of the patients admitted with heart failure was found to be 41.9 years (standard deviation 24.0 and median 41.5), the mean hospitalisation time was found to be 8.3 days (standard deviation 6.8 and median 6.5) and the most affected age group was found to be 30 - 49 years (31.4 of the total cases) as indicated in Table 2. There was also a worrisome significant number of young children ( 0 - 9 years) affected by the disease, $10.6 \%$, as shown in Table 1, which is attributed to the advent of HIV/AIDS as most of them were born HIV positive.

\begin{tabular}{|ccc|}
\hline \multicolumn{3}{|c|}{ Table 1. Gender and age distribution among the cases. } \\
\hline Variable & Frequency & Percentage \\
\hline Gender & Total $(\mathrm{N}=385)$ & $\%$ \\
\hline Males & 162 & 42.1 \\
\hline Females & 223 & 57.9 \\
\hline AGE (years) & $\mathrm{N}=$ & $\%$ \\
\hline $0-9$ & 41 & 10.6 \\
$10-19$ & 38 & 10.0 \\
$20-29$ & 44 & 11.4 \\
$30-39$ & 60 & 15.6 \\
$40-49$ & 61 & 15.8 \\
$50-59$ & 39 & 10.1 \\
$60-69$ & 35 & 9.1 \\
$70-79$ & 45 & 11.7 \\
$80-89$ & 19 & 4.9 \\
$90-99$ & 3 & 0.8 \\
\hline
\end{tabular}

Table 2. The mean age and mean hospitalisation times.

\begin{tabular}{ccccccccccc}
\hline Variable & Obs & Total & Mean & SD & $\min$ & $25 \%$ & Median & $75 \%$ & $\max$ \\
\hline Age (years) & 385 & 116118 & 41.9 & 24.0 & 0.2 & 24.5 & 41.5 & 62.5 & 92 \\
Hosp. time (days) & 385 & 3186 & 8.3 & 6.8 & 1.0 & 4.0 & 6.5 & 10.5 & 50 \\
\hline
\end{tabular}

*Obs is the number of observed records, "total" represents the age of all the patients put together and the sum-mation of hospitalisation time in days; and SD is the standard deviation. 
Table 3 highlights the following: Hypertension was diagnosed in almost half of the patients, with 189 patients (49.1\%) being confirmed to be hypertensive. There were 59 cases (15.3\%) diagnosed of diabetes, 40 (10.4\%) were found to be in renal failure, 35 patients $(9.1 \%)$ had hypercholesteremia, pneumonia accounting for 35 cases (9.1\%) as well and 20 patients (5.2\%) were confirmed to have Left Ventricular Dysfunction, LVD. There were 37 cases of anaemia, 12 (3.1\%) of which were diagnosed as Zidovudine, AZT, induced. Cardiomyopathy, CM, was also present in a number of patients, with 78 cases being recorded, $25(6.5 \%)$ of which were confirmed as HIV induced and $22(5.7 \%)$ confirmed as post-partum. There were also 4 (1.6\%) cases of thyrotoxicosis, 11 (2.9\%) cases of infective endocarditis, 10 (2.6\%) cases of Atrial Fibrillation, AF, 13 (3.4\%) cases with rheumatic heart disease, 34 (8.8\%) with Asthma/Chronic Obstructive Pulmonary Disease (COPD) and 15 (3.9\%) patients diagnosed of pericarditis.

As indicated in Table 4, almost all the patients admitted at the hospital and diagnosed of heart failure where prescribed a diuretic, furosemide, 379 (98.4\%). A small number of patients, $36(9.4 \%)$, were prescribed beta blockers. A large number of patients, 309 (80.3\%), were prescribed an ACEI. There were 192 (49.9\%) cases were prescribed spironolactone and 122 (31.7\%) digoxin. Quite a number of patients, 202 (52.5\%) were also prescribed antibiotics whilst 64 (16.6\%) patients were prescribed analgesics.

Of the 385 patients that were studied, 96 (24.9\%) died whilst there were 287 (74.5\%) normal hospital discharges due to the patients stabilising and responding well to treatment and one patient absconded whilst another one was discharged on care giver's request, as shown in Table 5. Of those who died, eight (8) were on beta blockers.

Table 3. Disease conditions associated with heart failure/concomitant diagnosis.

\begin{tabular}{|c|c|c|}
\hline Variable & $\begin{array}{l}\text { Frequency } \\
\qquad(\mathrm{N}=)\end{array}$ & $\begin{array}{c}\text { Percentage } \\
\text { (\%) }\end{array}$ \\
\hline Hypertension & 189 & 49.1 \\
\hline Diabetes & 59 & 15.3 \\
\hline Hypercholesteremia & 35 & 9.1 \\
\hline Pneumonia & 35 & 9.1 \\
\hline Rheumatic heart disease & 13 & 3.4 \\
\hline Infective endocarditis & 11 & 2.9 \\
\hline Asthma/COPD & 34 & 8.8 \\
\hline LVD & 20 & 5.2 \\
\hline $\mathrm{AF}$ & 10 & 2.6 \\
\hline Congenital heart disease & 20 & 5.2 \\
\hline Alcoholism & 4 & 1.0 \\
\hline Anaemia & 25 & 6.5 \\
\hline AZT-induced anaemia & 12 & 3.1 \\
\hline Cardiomyopathy & 31 & 8.1 \\
\hline HIV-induced CM & 25 & 6.5 \\
\hline Post-partum CM & 22 & 5.7 \\
\hline Thyrotoxicosis & 6 & 1.6 \\
\hline Pericarditis & 15 & 3.9 \\
\hline Renal Failure & 40 & 10.4 \\
\hline
\end{tabular}


Table 4. Medication/categories of medicines taken by the study patients.

\begin{tabular}{ccc}
\hline Type/class of drug & $\begin{array}{c}\text { Number of patients } \\
(\mathrm{N}=)\end{array}$ & $\begin{array}{c}\text { Percentage } \\
(\%)\end{array}$ \\
\hline Furosemide & 379 & 98.4 \\
Digoxin & 122 & 31.7 \\
ACEI & 309 & 80.3 \\
Spironolactone & 192 & 49.9 \\
Analgesics & 64 & 16.6 \\
Antibiotics & 202 & 52.5 \\
Beta blockers & 36 & 9.4 \\
\hline
\end{tabular}

Table 5. Cardiovascular (CVS) examination and patient discharge from hospital.

\begin{tabular}{ccc}
\hline CVS exam & $\mathrm{N}=$ & $\%$ \\
\hline ECHO & 317 & 82.3 \\
ECG & 315 & 81.8 \\
\hline Reason for hospital discharge & $\mathrm{N}=$ & $\%$ \\
\hline Stable, home on treatment & 287 & 74.5 \\
Absconded & 1 & 0.3 \\
Care giver's request & 1 & 0.3 \\
Died & 96 & 24.9 \\
\hline
\end{tabular}

\section{New Serology Screen}

Of all the 385 Congestive Heart Failure, CHF, admissions, 89 (23.1\%) patients were confirmed to be HIV negative, 86 (22.3\%) patients were confirmed as HIV positive whereas the HIV status of 210 (54.5\%) patients was not confirmed, as shown in Table 6.

Table 7 and Graph 1 show that there was a small number of beta blocker prescriptions observed. Atenolol was the most prescribed followed by propranolol and lastly carvedilol. Metoprolol and bisoprolol were not prescribed to any patient

The results shown in Table 8 indicate that before hospitalisation, 22 (5.7\%) patients were already taking beta blockers and after hospitalisation there were 36 (9.4\%) cardiac failure patients taking beta blockers. This implies that there were 14 (3.6\%) patients who were introduced to beta blocker therapy for the first time while in hospital. In patients who took beta blockers, 8 (22.2\%) died and 28 (77.8\%) were stabilised and discharged home. Of the patients who died whilst taking beta blockers, four were on propranolol, three were taking atenolol and one was on carvedilol.

The prevalence of cardiac failure hospitalisation for the year 2011 was $1.54 \%$ as shown in Table 9 .

The variables that were found to be statistically related to the prescription outcome include age ( $p<0.0001)$, hypertension $(\mathrm{p}<0.01)$, diabetes $(\mathrm{p}<0.0001)$ and renal failure $(\mathrm{p}<0.05)$.

No treatment variables were found to be statistically related to the occurrence of death.

Age showed a positive effect on the prescribing pattern of beta blockers, (older aged are more likely to be occupied), also diabetes showed a positive effect with beta blocker prescriptions (diabetics much more likely to be prescribed beta blockers), as indicated in Table 10 .

This is the minimal adequate model. 
Table 6. HIV status.

\begin{tabular}{ccc}
\hline HIV+ & Frequency & Percentage \\
\hline No & 89 & 22.1 \\
Yes & 86 & 22.3 \\
Not-confirmed & 210 & 54.6 \\
Total & 385 & 100.0 \\
\hline
\end{tabular}

Table 7. Beta blockers prescribed in the heart failure cases.

\begin{tabular}{ccc}
\hline Beta blocker & $\begin{array}{r}\text { Cases }(\mathrm{n}=) \\
(\text { total } \mathrm{n}=43)\end{array}$ & $\begin{array}{c}\text { Percentage } \\
(\%)\end{array}$ \\
\hline Atenolol & 30 & 7.8 \\
Propranolol & 7 & 1.8 \\
Carvedilol & 6 & 1.6 \\
Metoprolol & 0 & 0 \\
Bisoprolol & 0 & 0 \\
\hline
\end{tabular}

Table 8. Beta blocker prescriptions before and after hospitalisation.

\begin{tabular}{ccc}
\hline Beta blocker prescription & Frequency & Percentage \\
\hline Prior to admission & 22 & 5.7 \\
After admission & 36 & 9.4 \\
New BB patients & 14 & 3.6 \\
\hline
\end{tabular}

Table 9. Prevalence of heart failure hospitalisation for the year 2011.

\begin{tabular}{cc}
\hline Number of CHF hospitalisations & 317 \\
Total number of hospitalisation & 20,522 \\
Prevalence & $1.54 \%$ \\
\hline
\end{tabular}

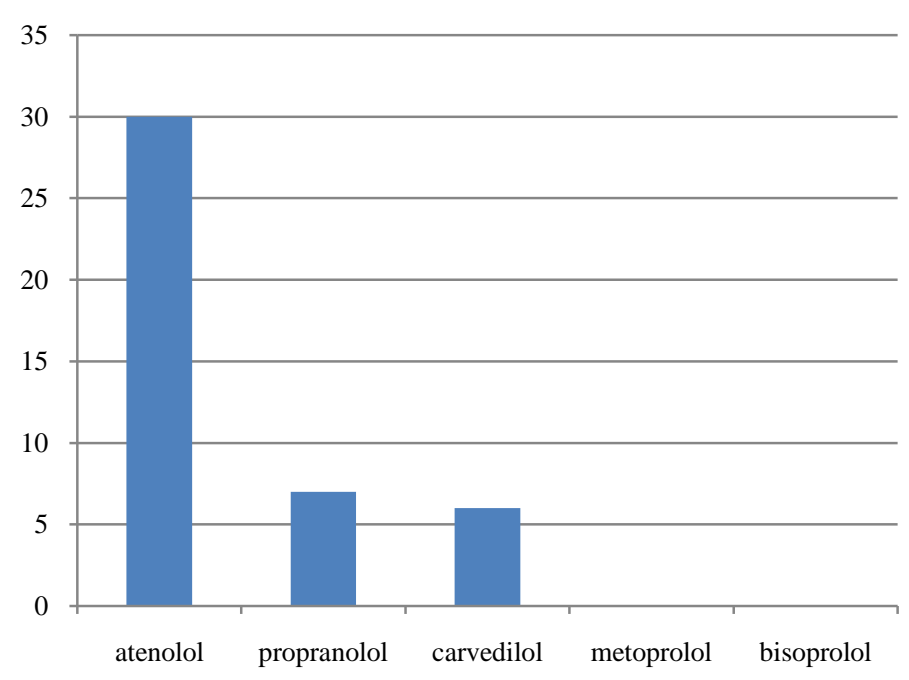

Graph 1. Beta blockers prescribed in the cardiac failure cases. 
Table 10. Relationship between variables (odds ratios).

\begin{tabular}{ccc}
\hline Variable & Odds Ratio (OR) & p-Value \\
\hline Beta blocker and increased age & 1.02 & 0.0107 \\
Beta blocker and diabetes & 2.22 & 0.0281 \\
Atenolol \& hypercholesterolemia & 5.56 & 0.000136 \\
Atenolol and hypertension & 5.01 & 0.004759 \\
Propranolol and age & 1.04 & 0.0332 \\
\hline
\end{tabular}

$$
\text { Logit [odds] }=-2.77+0.02 \mathrm{AGE}+0.80 \mathrm{DIABETES}
$$

The odds of prescribing beta blockers are 1.02 times for a year older individual. The odds of prescribing beta blockers are 2.22 times among the diabetics than the non-diabetic. The observations revealed an association between atenolol and hypertension and hypercholesterolemia, with ORs of $5.01(\mathrm{p}=0.004759)$ and $5.56(\mathrm{p}=$ $0.000136)$ respectively. Also, propranolol was demonstrated to be associated to age $(\mathrm{OR}=1.04, \mathrm{p}=0.0332)$. Diabetes was also associated with a beta blocker prescription.

\section{Discussion}

The results show that there were few beta blocker prescriptions by doctors in cardiac failure patients. This could have been due to the fact that most patients present to the hospital with CHF when the disease is at an advanced stage and clinicians tend to shun beta blockers because of the belief that beta blockers worsen heart failure especially in the frail and elderly patients [16] [17]. This reluctance to prescribe beta blockers in CHF by clinicians has been a matter of concern as it may deny patients of life-saving treatment, especially as they reverse modelling. Of the small number of beta blocker prescription observed, atenolol was the most prescribed followed by propranolol and lastly carvedilol. Metoprolol and bisoprolol were not prescribed to any patient. This trend could be because of the local national guidelines which recommend atenolol and propranolol as the beta blockers to use in CHF. Consequently, there haven't been much strides or effort by the practitioners in trying to introduce patients to the more efficacious beta blockers such as carvedilol, metoprolol and bisoprolol [18], as seen by the very few prescriptions of carvedilol and none on metoprolol and bisoprolol. The other factor of the low prescriptions could be related to the availability of the drugs in the hospital which can influence prescriptions of the readily available agents. These results do not compare well with international guidelines that recommend use of clinically proven beta blockers, carvedilol, metoprolol and bisoprolol, in CHF patients. Nevertheless, a significant number of patients who got prescribed beta blockers received protection from the disease and managed to recover and got discharged. Introduction of more efficacious beta blocker agents in the national treatment guidelines (EDLIZ) and prescribing to more chronic heart failure patients could further improve clinical care and significantly reduce mortality, hospitalisation time and symptoms; and improve the patients' quality of life.

There seem to be a gender shift in those affected by CHF. More men than women used to be reported as suffering from CHF but the results showed that more women (57.9\%) were affected by the condition than men (42.1\%) at the study hospital. This could be due to complications during pregnancies, which can precipitate heart failure, because of early marriages and the HIV infection, more women taking up stressful jobs initially reserved for men and lifestyle changes that have resulted in more women engaging in health-hazard practices such as smoking and beer-drinking.

The study showed that the main contributors to the diagnosed cardiac failure were hypertension 49.1\% (189), diabetes 15.3\% (59), hypercholesterolemia 9.1\% (35), pneumonia 9.1\% (35), renal disease 10.4\% (10), asthma/ COPD 8.8\% (34), rheumatic heart disease 3.4\% (13), LVD 5.2\% (20), congenital heart disease 5.2\% (20), anaemia 9.6 (37), cardiomyopathy 20.3\% (78) and pericarditis 3.9\% (15). These results are comparable to other studies concluded in sub-Saharan African. In the Heart of Soweto study, carried out in South Africa, the mean age of those with heart failure was 55 years and 57\% were women [19]. The gender proportion of those with heart failure in South Africa's Soweto suburb is a mirror image of the Zimbabwe's situation at this major referral hospital. In the Soweto study, the most common diagnosis was hypertension (33\%), cardiomyopathy (28\%), 
right heart failure (27\%) and rheumatic heart disease [19]. In a study carried out at St Elizabeth Catholic general hospital in Cameroon, the average age for the heart failure cases was 42.5 years. The cardiac failure cases were attributed to valvulopathies (35\%), cardiomyopathies (32\%), hypertension (15\%), pericarditis (7\%), COPD (8\%) and congenital heart disease (3\%) [20]. The Cameroon results are also comparable to this hospital study results. The notable similarities being the mean age, congenital heart disease, COPD and cardiomyopathies. The only major difference is that whilst in Zimbabwe's setting hypertension is the major contributor to heart failure with 49.1\%, in Cameroon it only contributes $15 \%$. This could be a genetic difference and/or lifestyle difference.

The results also confirm that hypertension, cardiomyopathy, rheumatic heart disease, chronic lung disease and pericardial disease account for over $90 \%$ of the heart failure cases in sub-Saharan Africa.

The prevalence of chronic heart failure hospitalisation at this studyhospital was found to be $1.54 \%$. The actual prevalence of heart failure cases is likely to be higher than the hospitalisation prevalence due to a number of factors such as more patients seeking medical help from primary health centres, some patients not affording to seek any medical help and some patients seeking alternative help (traditional or spiritual). According to some studies done, the prevalence of chronic heart failure is on the rise. This has been attributed to consequences of urbanisation, diet, lifestyle modification and risk-prone behaviour [13].

HIV seems to be contributing to the rise in heart failure cases. From the results, there were 12 cases (3.1\%) of AZT induced anaemia which subsequently led to heart failure. HIV-induced cardiomyopathy was a bit higher than the anaemia caused by AZT, having 6.5\% (25) cases. This can be summed up as having HIV contributing at least $9.6 \%$ of the heart failure cases. There were $36(9.4 \%)$ patients who were prescribed beta blockers, 14 patients taking a beta blocker for the first time. This was low as compared to other countries, especially the western world where they have embraced the importance of beta blockers in chronic heart failure [17]. Of the prescribed beta blockers, atenolol was the highly prescribed with a frequency of $30(7.8 \%)$ followed by propranolol with 7 patients (1.8\%) and carvedilol prescribed to 6 patients (1.6\%). No patient was prescribed metoprolol or bisoprolol. This is in contrast with international guidelines where beta blockers are part of the first line drugs in heart failure management [21] with carvedilol, bisoprolol and metoprolol amongst the recommended beta blocker drugs whilst atenolol and propranolol are not indicated for heart failure treatment [10]. This is an indication that the hospital prescribing patterns are not in tandem with evidence-based practice in heart failure management using beta blockers.

However, the results went on to show that furosemide and an ACEI (captopril/enalapril) were highly prescribed. This combination is used as the standard treatment at the hospital. In testing for an association between the standard treatment plus a beta blocker and death as the outcome; and standard treatment plus other drugs (digoxin or spironolactone or both) and death, it was discovered that there was no association between treatment combination and occurrence of death. This implies that, from the study, no treatment variables were found to be statistically related to the occurrence of death. There was also no statistical association between diseases presented and prescription of metoprolol, bisoprolol and carverdilol. Associations were however found between beta blocker prescription and age, $\mathrm{OR}=1.02(\mathrm{p}=0.0107)$, beta blocker prescription and diabetes, $\mathrm{OR}=2.22(\mathrm{p}$ $=0.0281)$, propranolol and age, $\mathrm{OR}=1.04(\mathrm{p}=0.0332)$, atenolol and hypercholesteremia, $\mathrm{OR}=5.56(\mathrm{p}=$ 0.000136), atenolol and hypertension, $\mathrm{OR}=5.01(\mathrm{p}=0.004759)$. From the odds ratio results, older people were more likely to be prescribed a beta blocker - the odds of prescribing a beta blocker were 1.02 times for a year older heart failure patient. The odds of prescribing a beta blocker were 2.22 times more for a diabetic heart failure patient than a non-diabetic heart failure patient. This has been elucidated in a study to find out if diabetes mellitus patients who are also heart failure patients benefit as well as the non-diabetic heart failure patients. It was found that the diabetes mellitus patients had a better prognostic benefit from beta blocker therapy than the non-diabetic heart failure patients [22]. Propranolol was found to be prescribed 1.04 times more per year increase in age, making it more likely to be prescribed in the elderly. Prescriptions of atenolol were demonstrated to increase with hypertensive and hypercholesteremic heart failure patients. In hypertension it is because atenolol has been proven to be effective in lowering blood pressure with less adverse effects than propranolol.

Echocardiography and ECG services were used regularly in the diagnosis of chronic heart failure as shown by the high number of patients with those examinations done for their diagnosis; ECHO (317) and ECG (315). In most cases, a patient would be subjected to both examinations. ECHO is regarded as the most accurate diagnostic tool for heart failure [23] and with its regular use at thehospital; patients are getting accurate diagnosis, provided the results are interpreted correctly.

The national treatment guidelines (EDLIZ) recommend the use of propranolol or atenolol under a specialist 
care when the standard treatment regimen plus other agents such as spironolactone and or digoxin have failed. This has helped in improving patients' outcomes but could be further improved. The guidelines are not up to date with the new evidence-based beta blockers such as bisoprolol, carvedilol and metoprolol recommended by international treatment guidelines like National Institute of Health Care Excellence (NICE). These relatively new beta blockers have been shown in various studies (for example CIBIS-II and MERIT-HF) to offer better prognosis in chronic heart failure patients. Thus, the inclusion of these newly recommended evidence-based agents in the EDLIZ could offer optimal therapy for the patients put on beta blockers and greatly improve their disease condition.

\section{Limitations}

The study was done in a public hospital setting only. Efforts to include a private hospital were unsuccessful as the authorities could not grant permission for the study to be carried out using their medical records. Time permitting; more hospitals across the country could have been included in the study to have a better picture of the health practice in the whole country. Also, a prospective study involving the outpatients department together with the retrospective one could have been the best study design, but the time limit of the study could not afford the prospective arm to be included as it required more time for completion.

\section{Conclusion}

There were more women than men diagnosed with cardiac failure at the hospital and the average age of CHF was forty two years. The prescribing rate of beta blockers was observed to be very low. Atenolol was the most prescribed beta blocker, followed by propranolol and lastly carvedilol. Metoprolol and bisoprolol were virtually not prescribed. The prevalence of chronic heart failure hospitalisation was $1.54 \%$. Hypertension, cardiomyopathy, renal failure, pneumonia, asthma/COPD, rheumatic heart disease, LVD, pericarditis and anaemia were the major contributors to cardiac failure. There is lack of evidence based prescribing of beta blockers in cardiac failure in the Zimbabwe's public health care system. The health care system is lagging behind in terms of implementing international guidelines on the use of beta blockers in chronic heart failure.

\section{Recommendations}

To have a better assessment of the prescribing patterns, more studies in other hospitals across the country have to be carried out. Treatment guidelines on the use of beta blockers in chronic heart failure should be constructed, in line with international guidelines, in order to create an evidence-based practice system. The involvement of all the critical stake holders that includes academia, research groups and the pharmaceutical industry, in the formulation of local guidelines is important so that results of all or most of the completed clinical studies and updated international guidelines are forwarded and discussed for possible consideration into the local guidelines. Of importance as well is the setting up of a special national health committee or consultancy that has the mandate of engaging the ministry of health, the pharmaceutical industry and health practitioners on the updates on evidence-based practice in line with recent clinical evidence or updates and also assessing how best the country's health care system could adopt or move with the changing times so as to afford the best possible treatment options to patients.

\section{Acknowledgements}

My heartfelt appreciation goes to the highly contributing Chairman of Clinical Pharmacology at the University of Zimbabwe, Prof CFB Nhachi, for his support and guidance. I greatly appreciate the advice extended to this work by Ms Celia Matyanga, who responded promptly and enthusiastically to my requests for frank comments, despite her congested schedule. I am indebted to all of them, who did their best to bring improvements through their contributions and suggestions. I am also thankful to my family, wife and kids, who directly or indirectly have been helpful in one way or the other. I thank my Dearest Parents for affording me the opportunity to explore life and extend my reach.

And to my late brother, Sylvester, you are always my greatest motivator, may your soul rest in peace.

To my wife and kids, I love you.

May God bless you. 


\section{References}

[1] Jay, N. (2000) Cardiac Remodelling-Concepts and Clinical Implications: A Consensus Paper from an International Forum on Cardiac Remodelling. JAAC, 45, 582-596.

[2] Packer, M., Carver, J.R., Rhodeheffer, R.J., et al. (1991) Effect of Oral Milrinone on Mortality in Severe Chronic Heart Failure. The New England Journal of Medicine, 325, 1468-1475. http://dx.doi.org/10.1056/NEJM199111213252103

[3] Digitalis Investigation Group (1997) The Effects of Digoxin on Mortality and Morbidity in Patients with Heart Failure. The New England Journal of Medicine, 336, 525-533. http://dx.doi.org/10.1056/NEJM199702203360801

[4] Massie, B.M., Fisher, S.G., Deedwania, P.C., Singh, B.N., Fletcher, R.D. and Singh, S.N., for the CHF-STAT Investigators (1996) Effect of Amiodarone on Clinical Status and Left Ventricular Function in Patients with Congestive Heart Failure. Circulation, 93, 2128-2134. http://dx.doi.org/10.1161/01.CIR.93.12.2128

[5] Pieske, B. (2004) Reverse Remodelling in Heart Failure-Fact or Fiction? European Heart Journal Supplements, 6, D66-D78. http://dx.doi.org/10.1016/j.ehjsup.2004.05.019

[6] Fletcher, P. (2000) Cardiovascular Medicines, John Hunter Hospital, Newcastle: Beta Blockers in Heart Failure. Australian Prescriber, 23, No 6.

[7] Hobbs, F.D.R. (2000) Management of Heart Failure: Evidence versus Practice. Does Current Prescribing Provide Optimal Treatment for Heart Failure Patients? British Journal of General Practice, 50, 735-742.

[8] Brophy, J.M., Joseph, L. and Rouleau, J.L. (2001) Beta Blockers in Congestive Heart Failure: A Bayesian MetaAnalysis. Annals of Internal Medicine, 134, 550-560. http://dx.doi.org/10.7326/0003-4819-134-7-200104030-00008

[9] Dobre, D., De Jongste, M.J., Lucas, C., Cleuren, G., van Veldhuisen, D.J., Ranchor, A.V. and Haaijer-Ruskamp, F. (2007) Effectiveness of Beta Blocker Therapy in Daily Practice Patients with Advanced Chronic Heart Failure: Is There an Effect-Modification by Age? British Journal of Clinical Pharmacology, 63, 356-364. http://dx.doi.org/10.1111/j.1365-2125.2006.02769.x

[10] McMurray, J.J., et al. (2012) ESC Guidelines for the Diagnosis and Treatment of Acute and Chronic Heart Failure. European Heart Journal, 33, 1787-1847.

[11] Eichhorn, E.J. and Bristow, M.R. (1996) Medical Therapy Can Improve the Biological Properties of the Chronically Failing Heart: A New Era in the Treatment of Heart Failure. Circulation, 94, 2285-2296.

[12] Shibata, M.C., Flather, M.D. and Wang, D.L. (2001) Systematic Review of the Impact of Beta Blockers on Mortality and Hospital Admissions in Heart Failure. European Journal of Heart Failure, 3, 351-357. http://dx.doi.org/10.1016/S1388-9842(01)00144-1

[13] Ntusi, N.B. and Mayosi, B.M. (2009) Epidemiology of Heart Failure in Sub-Saharan Africa. Expert Review of Cardiovascular Therapy, 7, 169-180.

[14] Hobbs, F.D., Jones, M.I., Allan, T.F., Wilson, S. and Tobias, R. (2000) European Survey of Primary Care Physician Perceptions on Heart Failure Diagnosis and Management. European Heart Journal, 21, 1877-1887. http://dx.doi.org/10.1053/euhj.2000.2170

[15] Magula, N.P. and Mayosi, B.M. (2003) Cardiac Involvement in HIV-Infected People Living in Africa: A Review. Cardiovascular Journal of Southern Africa, 14, 231-237.

[16] Berry, C., Murdoch, D.R. and McMurray, J.J.V. (2001) Economics of Chronic Heart Failure. European Journal of Heart Failure, 3, 283-291. http://dx.doi.org/10.1016/S1388-9842(01)00123-4

[17] Yilmaz, M.B., Refiker, M., Guray, Y., Guray, U., Altay, H., Dermirkan, B., Caldir, V. and Korkmaz, S. (2007) Prescribing Patterns in Patients with Systolic Heart Failure at Hospital Discharge: Why Beta Blockers Are Under-Prescribed or Prescribed at Low Dose in Real Life. International Journal of Clinical Practice, 61, 225-230. http://dx.doi.org/10.1111/j.1742-1241.2006.01157.x

[18] Krum, H. (1999) Beta Blockers in Heart Failure. The “New Wave” of Clinical Trials. Drugs, 58, 203-210. http://dx.doi.org/10.2165/00003495-199958020-00001

[19] Stewart, S., Wilkinson, D., Hansen, C., Vaghela, V., Mvungi, R., McMurray, J. and Sliwa, K. (2008) Predominance of Heart Failure in the Heart of Soweto Study Cohort. Emerging Challenges for Urban African Communities. Journal of American Heart Association, 118, 2360-2367. http://dx.doi.org/10.1161/circulationaha.108.786244

[20] Yancy, C.W., Fowler, M.B., Colucci, W.S., Gilbert, E.M., Bristow, M.R., Cohn, J.N., Lukas, M.A., Young, S.T. and Parker, M. (2001) Race and Response to Adrenergic Blockade with Carvedilol in Patients with Chronic Heart Failure. New England Journal of Medicine, 334, 1358-1365. http://dx.doi.org/10.1056/NEJM200105033441803

[21] NICE5 Guidelines, CG5. Chronic Heart Failure; Management of Chronic Heart Failure in Adults in Primary and Secondary Care, July 2003. www.nice.org.uk/guidance/cg5

[22] Haas, S.J., Vos, T., Gilbert, R.E. and Krum, H. (2003) Are Beta Blockers as Efficacious in Patients with Diabetes Mel- 
litus as in Patients without Diabetes Mellitus Who Have Chronic Heart Failure? A Meta-Analysis of Large-Scale Clinical Trials. American Heart Journal, 146, 848-853. http://dx.doi.org/10.1016/S0002-8703(03)00403-4

[23] Fuat, A., Hungin, A.P.S. and Murphy, J.J. (2003) Barriers to Accurate Diagnosis and Effective Management of Heart Failure in Primary Care: Qualitative Study. BMJ, 326, 196.

http://dx.doi.org/10.1136/bmj.326.7382.196 